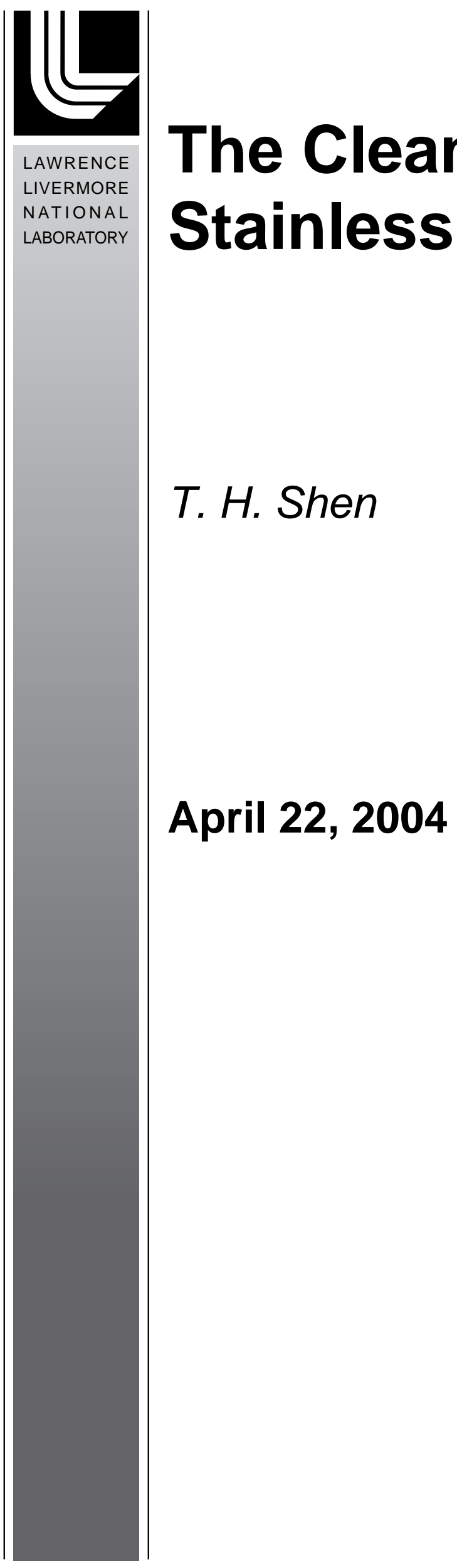


THIS DOCUMENT WAS PREPARED AS AN ACCOUNT OF WORK SPONSORED BY AN AGENCY OF THE UNITED STATES GOVERNMENT. NEITHER THE UNITED STATES GOVERNMENT NOR THE UNIVERSITY OF CALIFORNIA NOR ANY OF THEIR EMPLOYEES, MAKES ANY WARRANTY, EXPRESS OR IMPLIED, OR ASSUMES ANY LEGAL LIABILITY OR RESPONSIBILITY FOR THE ACCURACY, COMPLETENESS, OR USEFULNESS OF ANY INFORMATION, APPARATUS, PRODUCT, OR PROCESS DISCLOSED, OR REPRESENTS THAT ITS USE WOULD NOT INFRINGE PRIVATELY OWNED RIGHTS. REFERENCE HEREIN TO ANY SPECIFIC COMMERCIAL PRODUCT, PROCESS, OR SERVICE BY TRADE NAME, TRADEMARK, MANUFACTURER, OR OTHERWISE, DOES NOT NECESSARILY CONSTITUTE OR IMPLY ITS ENDORSEMENT, RECOMMENDATION, OR FAVORING BY THE UNITED STATES GOVERNMENT OR THE UNIVERSITY OF CALIFORNIA. THE VIEWS AND OPINIONS OF AUTHORS EXPRESSED HEREIN DO NOT NECESSARILY STATE OR REFLECT THOSE OF THE UNITED STATES GOVERNMENT OR THE UNIVERSITY OF CALIFORNIA, AND SHALL NOT BE USED FOR ADVERTISING OR PRODUCT ENDORSEMENT PURPOSES.

THIS WORK WAS PERFORMED UNDER THE AUSPICES OF THE U.S. DEPARTMENT OF ENERGY BY UNIVERSITY OF CALIFORNIA, LAWRENCE LIVERMORE NATIONAL LABORATORY UNDER CONTRACT W-7405-ENG-48. 


\title{
The Cleaning of 303 Stainless Steel
}

\author{
Tien H. Shen \\ Materials Science and Technology Division \\ Chemistry \& Materials Science Directorate
}

\begin{abstract}
The sulfur found on the surfaces of stainless steel 303 (SS303) after nitric acid passivation originated from the MnS inclusions in the steel. The nitric acid attacked and dissolved these MnS inclusions, and redeposited micron-sized elemental sulfur particles back to the surface. To develop an alternative passivation procedure for SS303, citric and phosphoric acids have been evaluated. The experimental results show neither acid causes a significant amount of sulfur deposit. Thus, these two acids can be used as alternatives to nitric acid passivation for NIF applications. For SS303 previously passivated by nitric acid, $\mathrm{NaOH}$ soak can be used as a remedial cleaning process to effectively remove the sulfur deposits.
\end{abstract}

$* * * * * * * * * * * * * * * * * * * * * * * * * * * * * * * * * * * *$

\section{INTRODUCTION}

In November 2001, the kinematic mounts made from 303 stainless steel (SS303) showed a high level of non-volatile residue (NVR) after nitric acid passivation followed by precision cleaning. The NVR, identified by John Ertel using Gas Chromatography/Mass Spectrometry (GC/MS), contained a high level of sulfur. Since sulfur is not permitted in the NIF laser beam environment, where equipment or parts (such as reflectors) may be silver coated, the presence of sulfur on the surface of SS303 components is a concern.

This investigation was initiated 1) to study the origin of sulfur on the surface of SS303 after nitric acid passivation, 2) to develop an alternative passivation and cleaning procedure and 3) to develop remedial cleaning procedure for SS303 parts that previously received the nitric acid passivation.

\section{The ORIGiN OF SULFUR}

The 303 stainless steel (sometime referred to as "free-machining" stainless steel) has chemical composition siihar to that of SS304 except that sulfur is intentionally added to 
form MnS inclusions in the microstructure. Table I shows the nominal chemical composition of these two alloys.

Table I The nominal chemical composition of stainless steels 303 and 304.

\begin{tabular}{|c|c|c|c|c|c|c|c|}
\hline Type & C & Mn & Si & Cr & Ni & P & S \\
\hline SS303 & 0.15 & 2.00 & 1.00 & $17.0-19.0$ & $8.0-10.0$ & 0.2 & $0.15 \mathrm{~min}$. \\
\hline SS304 & 0.08 & 2.00 & 1.00 & $18.0-20.0$ & $8.0-10.5$ & 0.045 & 0.03 \\
\hline
\end{tabular}

Figure 1 shows a typical microstructure of SS303. The MnS stringers help to promote the easy breakup of metal chips during machining.

It is likely that the sulfur found in the NVR measurements originated from the metal itself. To identify the origin of sulfur, an experiment was performed to simulate the hourlong nitric acid passivation followed by a precision cleaning with a mirror-polished SS303 sample. Figure 2a shows that the 35 vol\% $\%^{\dagger}$ nitric acid dissolved the MnS inclusions and left many pits behind. Micron-sized elemental sulfur particles, which were identified by EDXS/SEM as shown in Figure $\mathbf{2 b}$, were re-deposited back to the surface in the vicinity of these pits as indicated by arrows in Figure 2a. Apparently, the precision cleaning could not remove these sulfur particles.

A thermodynamic calculation ${ }^{1}$ suggested that $\mathrm{MnS}$ was thermodynamically unstable above a potential of $100 \mathrm{mV}^{\ddagger}$ and could not exist in solution with $\mathrm{pH}$ less than 4.3 . The strong oxidation potential of the nitric acid could dissolve the $\mathrm{MnS}$ and redeposit sulfur according to the reactions ${ }^{1,2,3}$ :

$$
\begin{aligned}
\mathrm{MnS}+2 \mathrm{H}^{+} & \rightarrow \mathrm{Mn}^{2+}+\mathrm{H}_{2} \mathrm{~S} \\
\mathrm{H}_{2} \mathrm{~S} & \rightarrow \mathrm{S}+2 \mathrm{H}^{+}+2 \mathrm{e}^{-}
\end{aligned}
$$

The observation of the elemental sulfur particles is consistent with these reactions.

The precision cleaning that followed the nitric acid passivation could not remove these sulfur deposits from the surface as shown in Figure 2b. Thus, it is likely that the sulfur found in the NVR measurements was generated by the nitric acid during the passivation process.

\section{Experimental Procedures}

To confirm this preliminary finding and develop an alternative to the nitric acid passivation, phosphoric and citric acids have been evaluated in this investigation. Furthermore, since some sulfur was apparently rinsed off by the Freon ${ }^{\mathrm{TM}}$ during the NVR measurements, this experiment also evaluated the effectiveness of using solvent to remove sulfur from the surface after the acid passivation.

\footnotetext{
$\dagger$ The 35 vol\% concentration is derived by diluting $50 \%$ of a 70 vol. $\%$ reagent grade nitric acid.

$¥$ Against Standard Hydrogen Electrode.
} 
Three 12"x12" SS303 plates with machined surface (roughness $\left(r_{a}\right) \leq 32 \mu$ inch) were used for this experiment. These plates were machined using NIF approved cutting fluid. The two sides of each plate were identified as $\mathbf{A}$ and $\mathbf{B}$.

The experimental procedures are outlined as follows:

1. Each plate was high pressure detergent washed with 5\% Brulin 1990 GD solution, and rinsed with high pressure spray of process water ${ }^{\S}$.

2. First NVR measurement was taken from Side A of each plate.

3. Each panel was treated with different acids as indicated below:

Plate 1): 50 vol\% nitric acid, 60 min., Plate 2): 15 vol\% citric acid gel, $120 \mathrm{~min}$., Plate 3): 15 vol\% phosphoric acid gel, $90 \mathrm{~min}$.

4. After the passivation, each plate was rinsed with high-pressure spray of process water.

5. A final wash of each plate with 5\% Brulin solution, and rinsed with high pressure spray of process water.

6. Second NVR and the first particle swipe were taken from Side A of each plate.

7. Soaked the test panels in acetone ${ }^{* *}$ for $10 \mathrm{~min}$., followed by hand-wiping of each plate with acetone.

8. The third NVR was taken followed by particle swipe from Sides A and $\mathbf{B}$, respectively, of each plate.

\section{RESULTS}

Table II shows the results of the experiment. This experiment again confirms the preliminary finding that the nitric acid passivation of SS303 (Plate 1) caused high NVR and high particle swipe values.

Table II The results of nitric, citric and phosphoric acid passivations on NVR and particle swipe.

\begin{tabular}{|c|c|c|c|c|c|c|}
\hline & \multicolumn{6}{|c|}{ "Results of NVR $\left(\mu \mathrm{g} / \mathrm{cm}^{2}\right) /$ Particle Swipe } \\
\hline & \multicolumn{2}{|c|}{ Plate 1} & \multicolumn{2}{|c|}{ Plate 2} & \multicolumn{2}{|c|}{$\begin{aligned} \text { Plate } 3 \\
\end{aligned}$} \\
\hline \multirow{2}{*}{$\begin{array}{c}\text { Cleaning and } \\
\text { Passivation Steps }\end{array}$} & \multicolumn{2}{|c|}{ Nitric (60 min.) } & \multicolumn{2}{|c|}{ Citric (120 min.) } & \multicolumn{2}{|c|}{ Phosphoric (90 $\mathrm{min}$.) } \\
\hline & Side A & Side B & Side A & Side B & Side A & Side B \\
\hline After 1st Brulin Wash & $0.52 /-$ & - & $0.46 /-$ & - & $0.49 /-$ & - \\
\hline Acid Passivation & - & - & - & - & - & - \\
\hline After 2nd Brulin Wash & $0.11 / 66$ & - & $0.01 / 69$ & - & $0.08 / 74$ & - \\
\hline Acetone Soak \& Wipe & $0.05 / 92$ & $0.14 / 86$ & $0.04 / 66$ & $0.02 / 75$ & $\mid 0.02 / 81$ & $0.03 / 74$ \\
\hline
\end{tabular}

$\S$ The process water was pressurized to $2,500 \mathrm{psi}$ and heated to $43-54{ }^{\circ} \mathrm{C}$. This condition applied to all the remaining procedures.

** We choose acetone since John Ertel had shown that the acetone could reduce sulfur of a 303 plate that failed previously of NVR. 
Both citric acid and phosphoric acid passivations reduced the NVR and particle swipe to an acceptable level of less than $0.1 \mu \mathrm{g} / \mathrm{cm}^{2}$ and level 83, respectively. Figure 3 shows the surface morphology of the SS303 after the citric acid and phosphoric acid passivations. Both acids attacked the MnS inclusions and left pits behind. However, the SEM investigation did not find any sulfur particles in the vicinity of these pits.

The acetone soak after the nitric-acid passivation was not consistently effective in removing the sulfur from the surface. Subsequently, several solvents, i.e. Freon ${ }^{\mathrm{TM}}$, isopropyl alcohol (IPA) and hot D.I. water, had also been evaluated for their effectiveness in removing the sulfur. However, the experiments showed erratic and inconsistent NVR results. Figure 4 shows that the hot D.I. water didn't noticeably dissolve the sulfur particles, but Freon did show some effectiveness in dissolving the sulfur particles. However, sulfur particles were still present after several Freon rinses.

\section{DisCUSSIONS}

In general, the primary purpose of passivation of stainless steel is to enhance its corrosion resistance and prevent the "rouging" 4 from occurring. In cleaning NIF parts, the passivation serves as part of the cleaning procedure to ensure the surfaces of stainless steel from contaminants, such as metal fines, welding debris, before the precision cleaning. In the case of SS303, the generation of sulfur during nitric acid passivation presents an additional problem. This study has shown that by replacing the nitric acid with citric or phosphoric acids during passivation, the problem of surface sulfur deposits can be effectively mitigated. However, the author cautions the reader that the citric and phosphoric acids are not effective passivation methods for SS303 for application in aggressive corrosion environment, such as salt containing atmosphere. This study has conducted a very extensive corrosion test in a salt spray environment on SS303 and SS304 passivated either by nitric, citric or phosphoric acids. These test showed that the citric acid passivated SS303 has poor corrosion performance compared to that of the nitric acid passivated SS303. However, from the corrosion point of view, the atmosphere inside the NIF beam line is rather benign and aggressive corrosion is not a concern. For the briefness of this report, these corrosion results are not presented and discussed here.

Remedial Cleaning Procedure For SS303 parts that previously received the nitric acid passivation, the experimental results show that solvents and hot D.I. water were not effective in removing the element sulfur. Hence, an alternate approach to removing sulfur was necessary.

It has been shown that the nitric acid is not effective in passivating free-machining stainless steels ${ }^{5}$. To properly passivate SS303, a NaOH soak after an nitric acid passivation is necessary to improve the corrosion resistance in a salt spray environment. It was suggested ${ }^{5}$ that residual nitric acid might be retained inside the pits after the passivation and degraded the corrosion resistance. The $\mathrm{NaOH}$ soak neutralized the retained nitric acid thus improving the corrosion resistance of SS303. To verify the effectiveness of $\mathrm{NaOH}$ soak, several experiments have been conducted to evaluate the 
corrosion performance of SS303 treated by $5 \mathrm{vol} \% \mathrm{NaOH}$ at $70^{\circ} \mathrm{C}$ for 30 minutes after the nitric acid passivation. The results showed that the corrosion performance was indeed improved with the $\mathrm{NaOH}$ soak. Figure 5 shows that the $\mathrm{NaOH}$ dissolved the sulfur particles, as indicated by arrows, and improved the pitting corrosion resistance of SS303 after the nitric acid passivation.

To pursue the $\mathrm{NaOH}$ soak as the remedial cleaning method for nitric-acid passivated SS303, a precision cleaning experiment was conducted on two 12" x 12" SS303 panels as follows:

1. Detergent-washed both panels with a high pressure $(2,500 \mathrm{psi})$ solution containing 5\% Brulin $1990 \mathrm{GD}$, heated to $43-54^{\circ} \mathrm{C}$ and rinse with high pressure spray of process water heated to $43-54^{\circ} \mathrm{C}$.

2. Passivated the two panels with $50 \%$ nitric acid for 60 min with Side A up follow by DI water rinse.

3. Soaked Panel 2 in $5 \% \mathrm{NaOH}$ at $70^{\circ} \mathrm{C}$ for 30 min. with Side A up, follow by DI rinse.

4. Detergent-washed both plates with a high pressure $(2,500 \mathrm{psi})$ solution containing $5 \%$ Brulin $1990 \mathrm{GD}$, heated to $43-54^{\circ} \mathrm{C}$ and rinsed with high pressure spray of process water heated to $43-54^{\circ} \mathrm{C}$.

5. Took NVR followed by particle swiping from Side A of each plate.

Table III shows the result of this cleaning experiment. The $\mathrm{NaOH}$ soak generated other type of compounds (not yet identified) on the surfaces of the sample and failed the NVR and particles swipe. However, the cleanliness can be improved by hand-wiping the $\mathrm{NaOH}$ treated SS303 with $100 \%$ IPA.

Table III The result of $\mathrm{NaOH}$ soak cleaning experiment.

\begin{tabular}{|c|c|c|}
\hline & Particle Swipe & NVR $\left(\mathrm{mg} / \mathrm{ft}^{2}\right)$ \\
\hline Panel 1 (Nitric passivated) & 71 & 0.13 \\
\hline Panel 2 (Nitric passivated + NaOH Soak) & 90 & 0.14 \\
\hline After hand-wipe with IPA & 65 & 0.02 \\
\hline
\end{tabular}

Based on this result, we recommend the use of $\mathrm{NaOH}$ soak followed by wiping with $100 \%$ IPA as the remedial cleaning procedure for SS303 parts that previously received the nitric acid passivation. However, for intricate parts which have surfaces that could not be easily reached by hand-wiping. The use of SS303 with nitric acid passivation followed by $\mathrm{NaOH}$ soak is not recommended. 


\section{CONCLUSION}

- The sulfur found on the surfaces of SS303 after the nitric acid passivation originated from the $\mathrm{MnS}$ inclusions in the steel. The nitric acid attacked and dissolved these $\mathrm{MnS}$ inclusions. Elemental sulfur was re-deposited back to the surface as either sulfur film or micron-sized particles. In addition, the pits left behind by the MnS inclusions were also filled with a high concentration of sulfur.

- Neither citric-acid nor phosphoric-acid passivation result in significant sulfur deposits after passivation. Thus, these two acids can be used as an alternative acid to passivate SS303.

- The $\mathrm{NaOH}$ soak after the acid passivation removes the sulfur deposits and significantly improves the pitting resistance of the acid-passivated SS303, but surfaces must be wiped with $100 \%$ IPA to meet NIF cleanliness requirements. Thus, we recommend the use of $\mathrm{NaOH}$ soak followed by wiping with $100 \%$ IPA as the remedial cleaning procedure for SS303 parts that previously received the nitric acid passivation.

\section{ACKNOWLEDGEMENTS}

The author would like to express his thanks to Ed Lindsey for conducting the SEMEDXS analysis. As always, the encouragements and suggestions offered by Bill Gourdin during the course of this investigation and the reviewing of this manuscript are also greatly appreciated.

\section{REFERENCES}

1. G. Eklund, "Initiation of pitting at sulfide inclusions in stainless steel", $J$. Electrochem. Soc 121(1974), pp461- 413.

2. G. Wranglén, "Pitting and sulphide inclusions in steel", Corrosion Sci. 14 (1974) pp 331-349.

3. J. Stewart and D. Williams, " The initiation of pitting corrosion on austenic stainless steel: on the role and importance of sulphide inclusions", Corrosion Sci. 33 (1992) pp 457-474.

4. R. Coreett, "Rouging - A discoloration of stainless steel surfaces", Materials Performance, Vol. 40 (2001), No. 2, pp 64- 66.

5. T. DeBold, "Passivating Stainless-Steel Parts", Machine and Tool Blue Book, vol. 81 (Nov. 1986), pp 74-77. 


\section{As-Polished Condition}

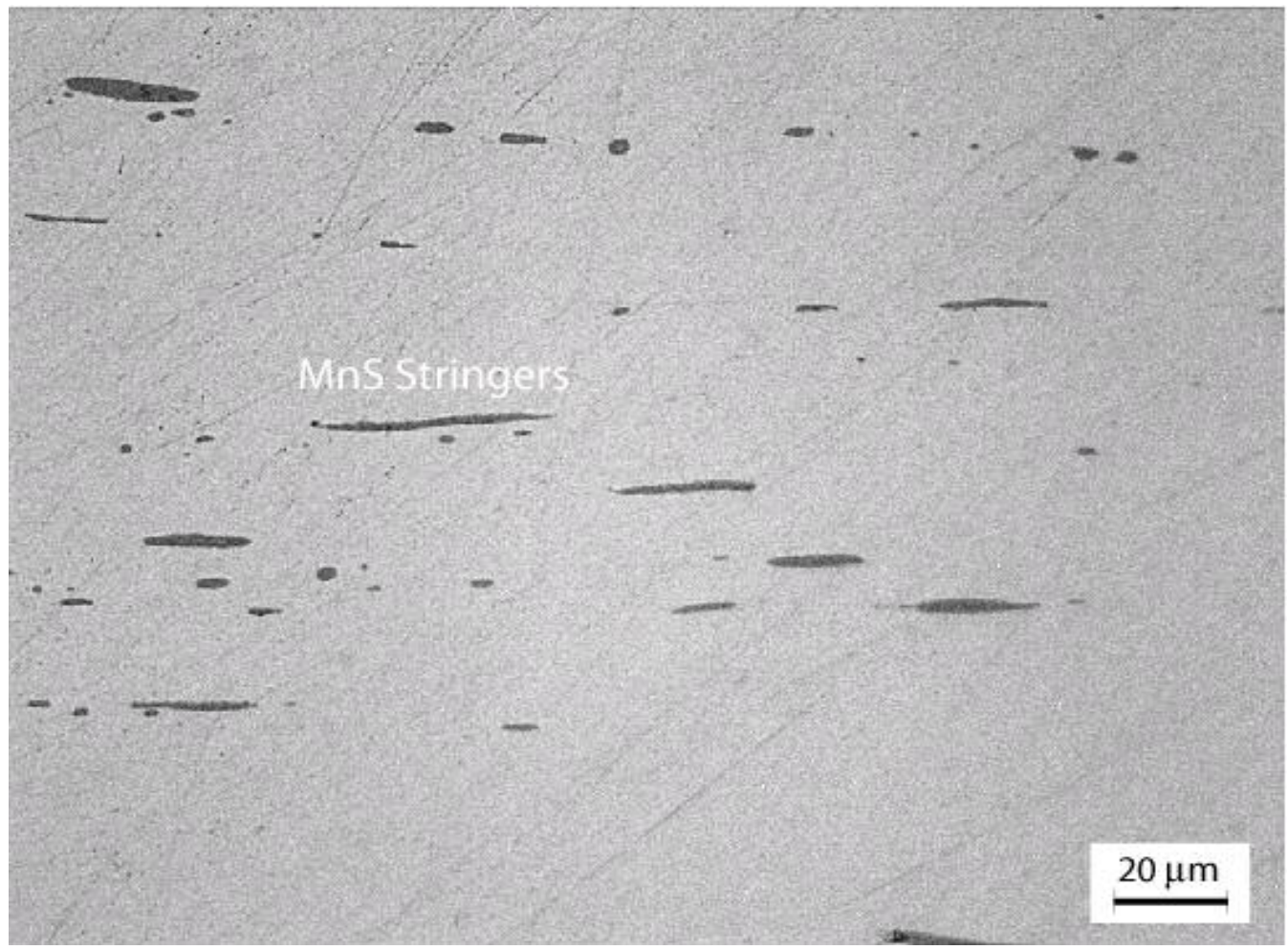

Figure 1 Typical microstructure of 303 stainless steel. The long stringers are $\mathrm{MnS}$ inclusions. 


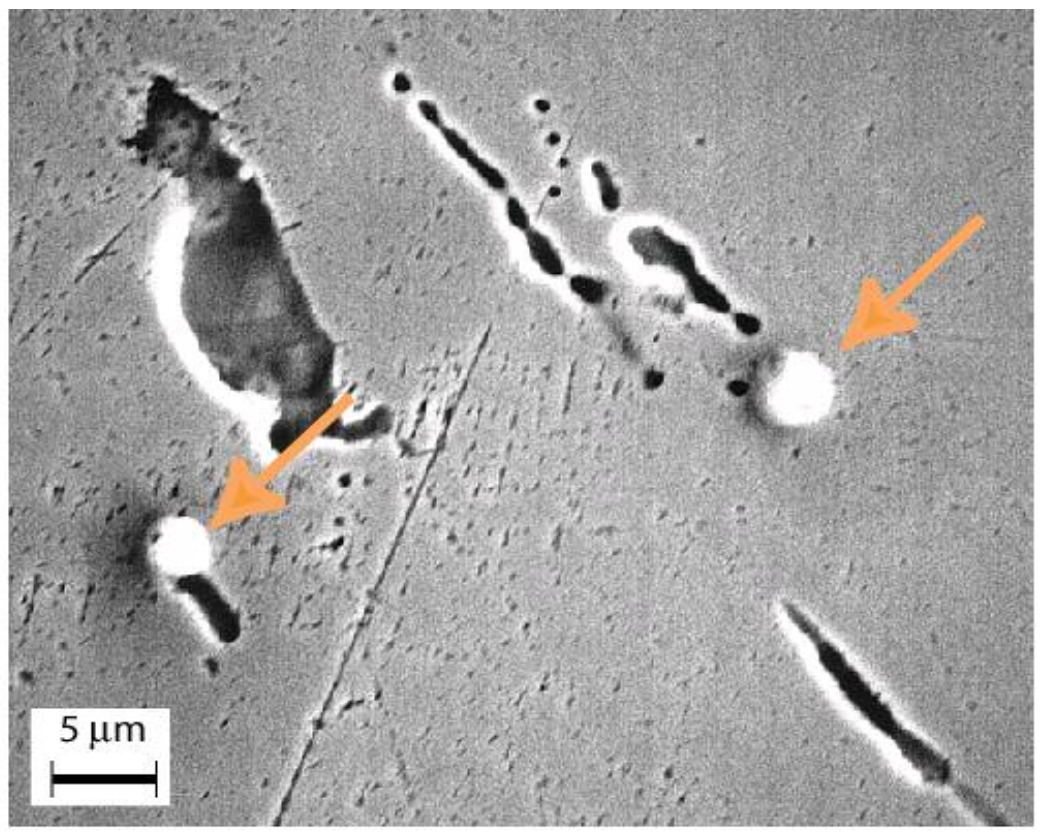

Fig 2a

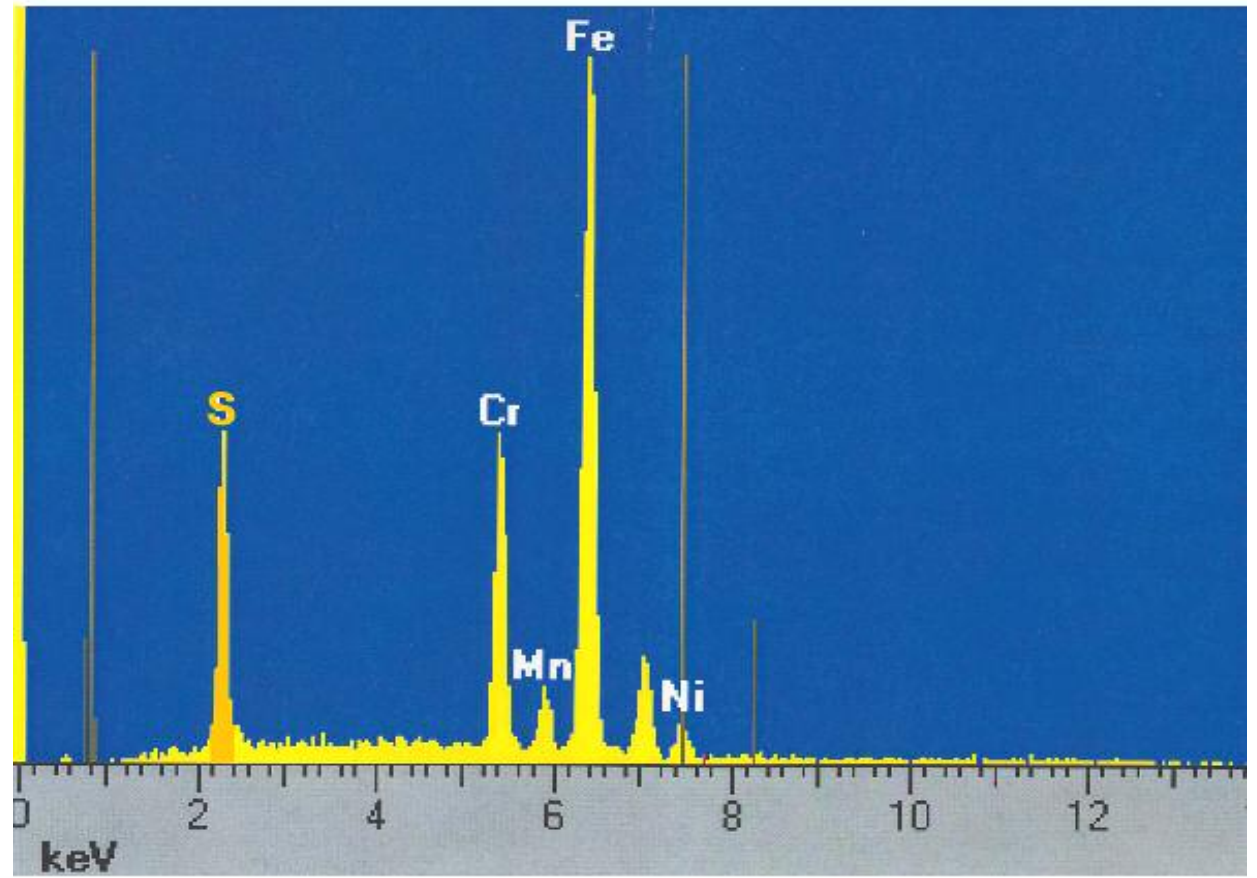

Fig 2b

Figure 2a shows that the nitric acid dissolved the MnS inclusions and left many pits behind. Micron-size elemental sulfur particles, identified by EDXS as shown in Figure 2b, were redeposited back to the surface in the vicinity of these pits as indicated in Figure 2a. The precision cleaning that followed the nitric-acid passivation could not remove these particles. 

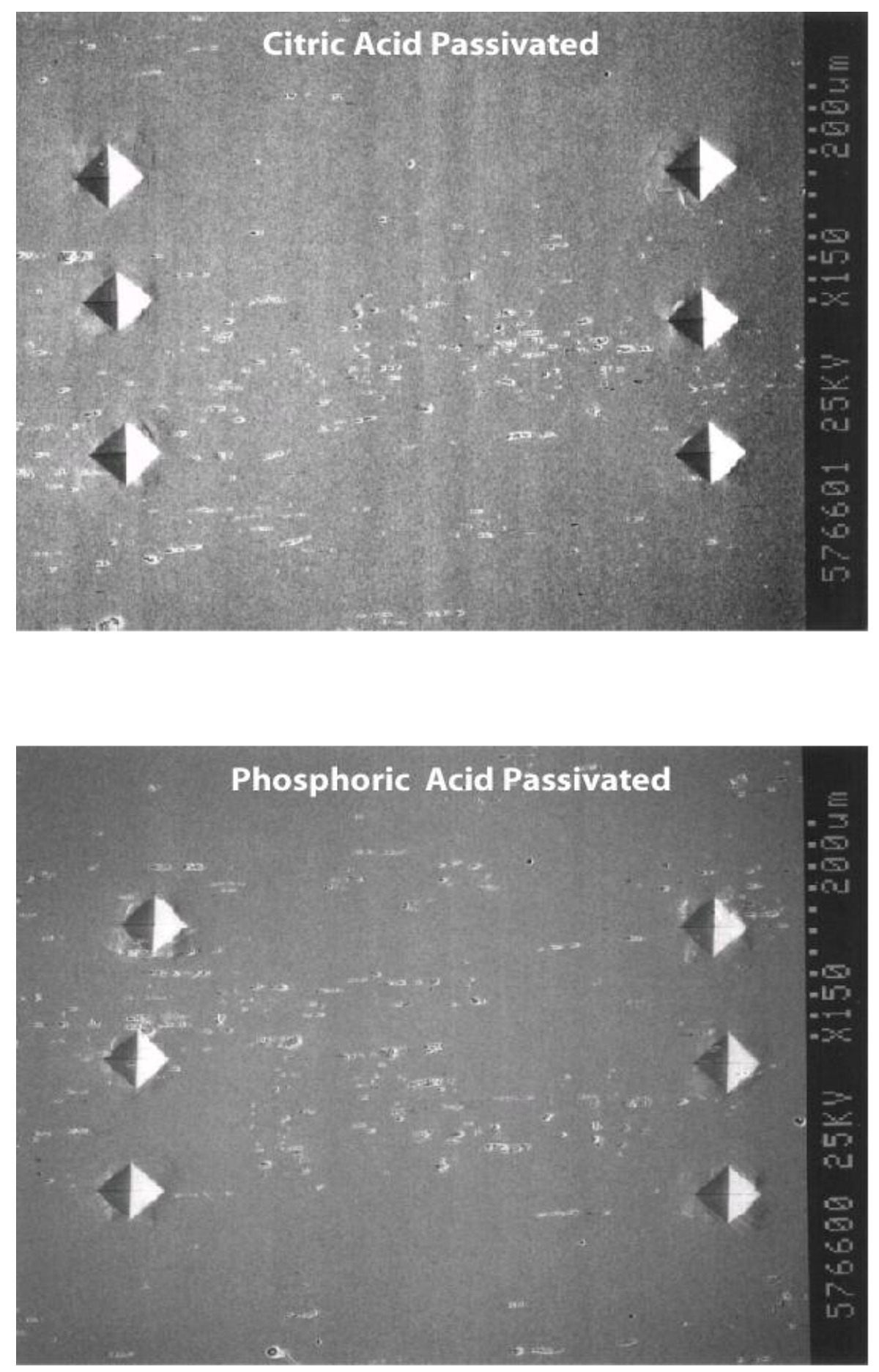

Figure 3 Both citric and phosphoric acids attacked the $\mathrm{MnS}$ inclusions and left pits behind. However, no elemental sulfur deposits were found in the vicinity of these pits. 
After 50 vol\% nitric acid passivation for $60 \mathrm{~min}$.

After 50 vol\% nitric acid passivation for $60 \mathrm{~min}$. t

Hot D.I. Water at $150^{\circ} \mathrm{F}$ for $10 \mathrm{~min}$.

After 50 vol\% nitric acid passivation for $60 \mathrm{~min}$.

\section{t}

Hot D.I. Water at $150^{\circ} \mathrm{F}$ for $10 \mathrm{~min}$.

\section{$\checkmark$}

Freon rinse
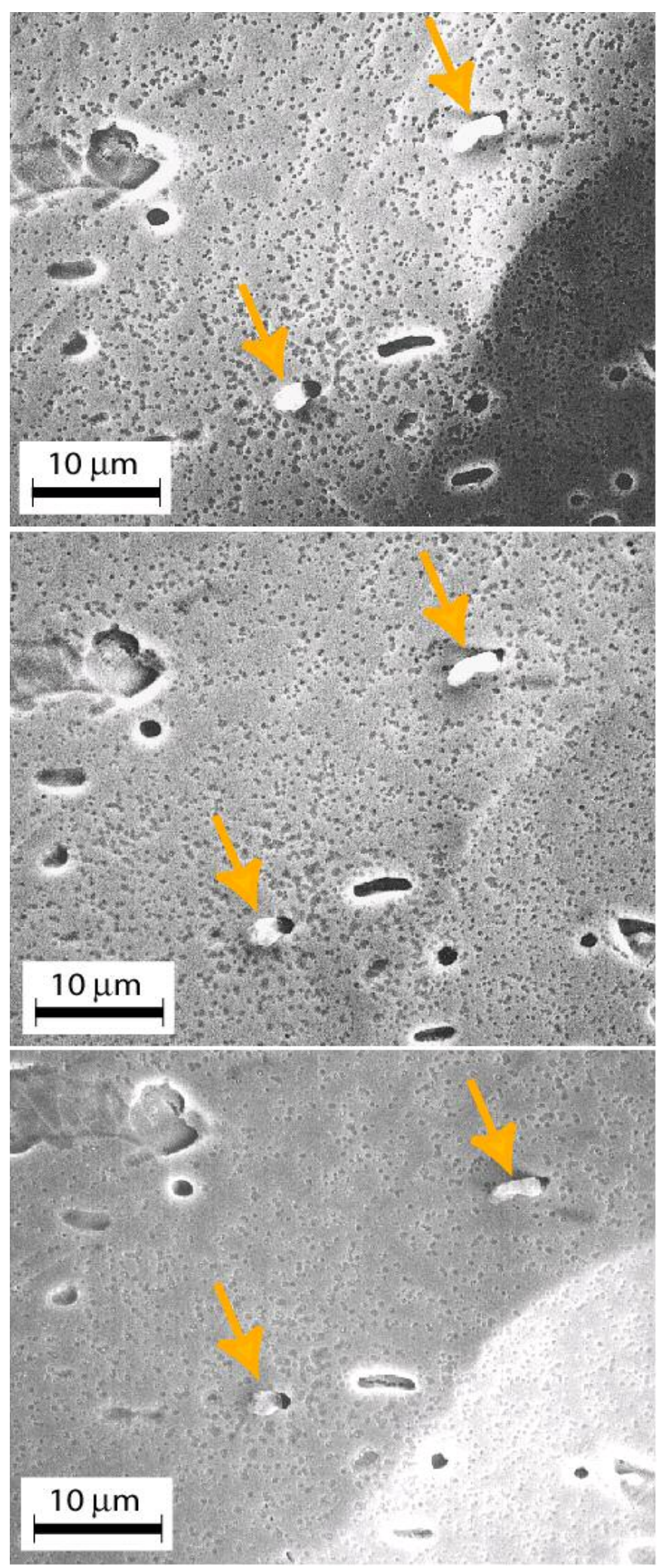

Figure 4 The sulfur particles, as indicated by arrows, didn't significantly dissolve by the hot DI water. However, Freon shows some capability in dissolving the sulfur. 


\section{After nitric acid passivation}

\section{Nitric acid passivation $+$ $\mathrm{NaOH}$ Neutralization}
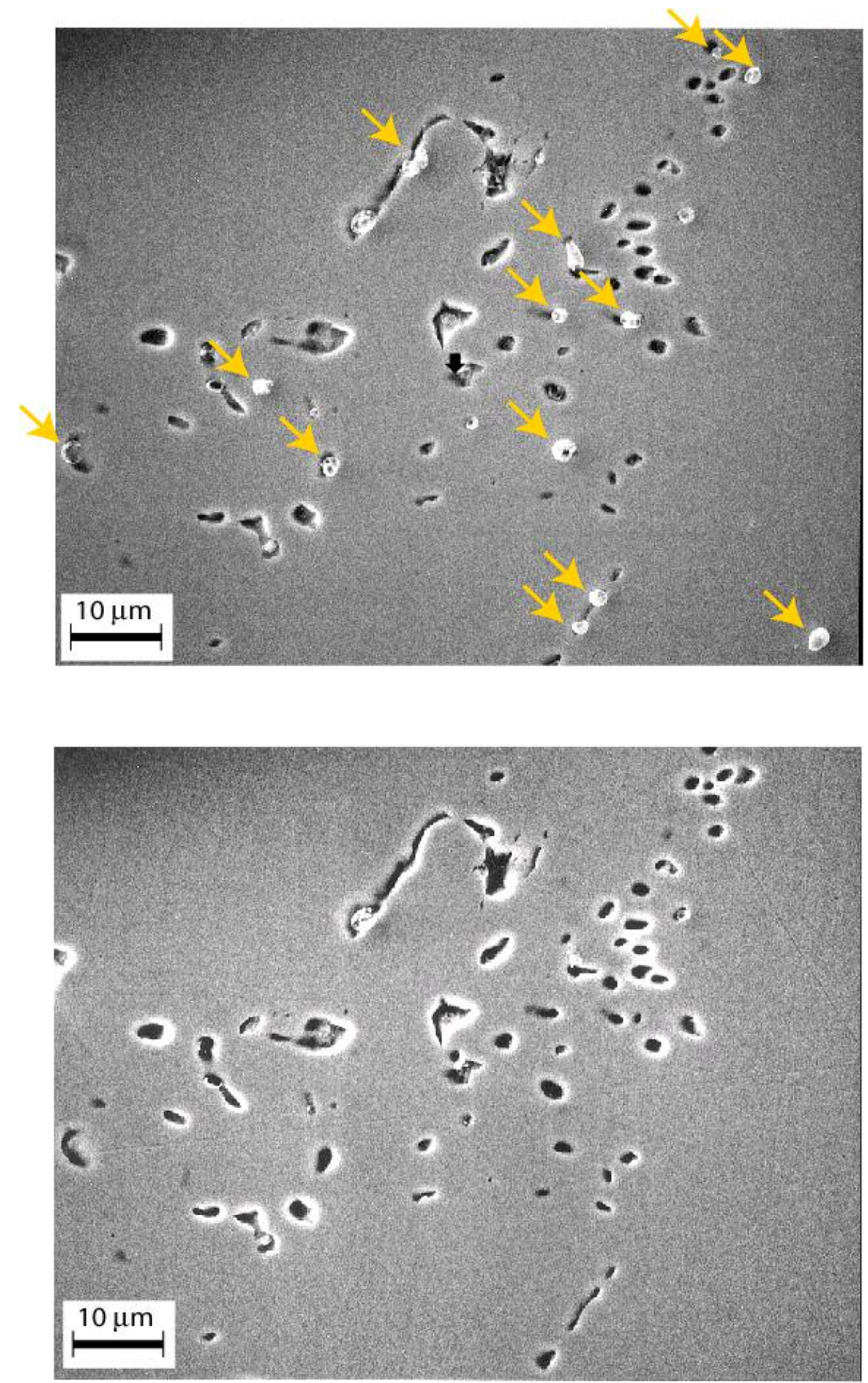

Figure 5 The $\mathrm{NaOH}$ dissolved the sulfur particles, as indicated by arrows, and improved the pitting corrosion resistance of SS303 after the nitric-acid passivation. 